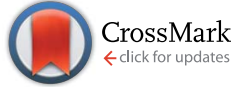

Cite this: Nanoscale, 2014, 6, 11953
Received 28th May 2014

Accepted 7th August 2014

DOI: $10.1039 / c 4 n r 02939 a$

www.rsc.org/nanoscale

\title{
Disentangling the effects of nanoscale structural variations on the light emission wavelength of single nano-emitters: InGaN/GaN multiquantum well nano-LEDs for a case study $\dagger$
}

\begin{abstract}
George Sarau, ${ }^{\text {*a }}$ Martin Heilmann, ${ }^{a}$ Michael Latzel $^{\mathrm{ab}}$ and Silke Christiansen ${ }^{\text {ac }}$
The scattering in the light emission wavelength of semiconductor nano-emitters assigned to nanoscale variations in strain, thickness, and composition is critical in current and novel nanotechnologies from highly efficient light sources to photovoltaics. Here, we present a correlated experimental and theoretical study of single nanorod light emitting diodes (nano-LEDs) based on InGaN/GaN multiquantum wells to separate the contributions of these intrinsic fluctuations. Cathodoluminescence measurements show that nano-LEDs with identical strain states probed by non-resonant micro-Raman spectroscopy can radiate light at different wavelengths. The deviations in the measured optical transitions agree very well with band profile calculations for quantum well thicknesses of 2.07-2.72 nm and In fractions of 17.5-19.5\% tightly enclosing the growth values. The nanorod surface roughness controls the appearance of surface optical phonon modes with direct implications on the design of phonon assisted nano-LED devices. This work establishes a new, simple, and powerful methodology for fundamental understanding as well as quantitative analysis of the strain - light emission relationship and surface-related phenomena in the emerging field of nano-emitters.
\end{abstract}

\section{Introduction}

Nano-emitters based on InGaN/GaN multiple quantum well (MQW) heterostructures that constitute the active components of nanorod light emitting diodes (nano-LEDs) are currently being explored not only as a viable solution to unravel longpending technological issues in solid state lighting such as inefficient light extraction, "efficiency droop", and "greenyellow gap"1-3 but also for novel and exciting applications in biosensing and bioimaging, ${ }^{4}$ nanolasers, ${ }^{5}$ subwavelength lithography, ${ }^{6}$ photovoltaics, ${ }^{7-9}$ and polarized single-photon emission. ${ }^{10}$ The characteristic properties of nano-LEDs as compared to their planar film counterparts include an ultraefficient and directed light out-coupling, a high internal quantum efficiency, and multicolor, white light emission even without additional tuning of the large area surfaces. These unique features have been achieved by choosing appropriate sizes and geometries as well as by controlling the strain

\footnotetext{
${ }^{a}$ Max Planck Institute for the Science of Light, Günther-Scharowsky-Str. 1, 91058 Erlangen, Germany.E-mail: george.sarau@mpl.mpg.de

${ }^{b}$ Institute of Optics, Information and Photonics, University of Erlangen-Nuremberg, Staudtstr. 7/B2, 91058 Erlangen, Germany

${ }^{c}$ Helmholtz Centre Berlin for Materials and Energy, Hahn-Meitner Platz 1, 14109 Berlin, Germany
}

$\dagger$ Electronic supplementary information (ESI) available. See DOI: 10.1039/c4nr02939a relaxation either through nanopatterning of planar films using top-down lithography techniques, ${ }^{1,11-14}$ or by bottom-up growth of relaxed InGaN/GaN MQWs on strain-free GaN nanowire templates. ${ }^{3,6,15-17}$

The key to wavelength-independent emission efficiency over the entire visible spectrum resides mainly in the control of the strain at the nanoscale. In InGaN/GaN MQW heterostructures there is a lattice mismatch between the two materials that can lead to a significant in-plane biaxial strain inside the QWs. This strain changes the crystal lattice geometry giving rise to two effects, namely the shift and bending of the energy bands. The latter effect, known as the piezoelectric effect, occurs because of the polar nature of the crystal structure along the $c$-axis of GaN and can induce a high internal electrostatic field that affects the overlap between the electron and hole wavefunctions in the quantum wells, referred to as the quantum confined Stark effect. ${ }^{18}$ Since the strain modifies the wavelength and intensity of the light emitted from the nano-emitter through locally confined changes in the band profile and spatial localization of the carrier wavefunctions, ${ }^{19}$ it is very important to quantitatively probe the strain state of single, isolated, and vertically aligned nanorod LEDs on the initial substrate as integrated in devices in order to assess the homogeneity and degree of the strain relaxation at the nanoscale compared to the starting film as a result of the nanofabrication process. Another essential point is the impact of intrinsic fluctuations in the thickness and In 
composition on the light emission wavelength from partially or fully strain relaxed nano-LEDs as compared to the bulk strained MQW counterparts. ${ }^{15,16,20,21}$ The high surface-to-volume ratio characteristic for the nanorod geometry leads to surface-related phonon modes whose interaction with electrons by way of Fröhlich phonon-electron coupling potentially open the way towards designing of phonon assisted nanorod LED devices. ${ }^{22,23}$ Therefore, such basic studies require the fabrication of reproducible nanostructures with small size deviations as well as controlled orientation and density.

In this context, it is obvious that a smart choice of the nanoemitter dimensions coupled with the development of a waferscale nanofabrication method minimizing the nanorod-tonanorod variations are critical for a fast and straightforward advancement in this field. It has been established that there is an optimum diameter for LED nanorods ranging from $100 \mathrm{~nm}$ to $200 \mathrm{~nm}$ at heights $\geq 1 \mu \mathrm{m}$ that warrants a highly efficient and collimated light emission towards the surface normal of up to $79 \%$ through the nanoscale control of the number and radiation pattern of optical modes as well as a good overlap between these modes and the active quantum wells. ${ }^{\mathbf{1 , 2 4}}$ Strain distribution simulations revealed that the decrease in the GaN nanorod diameter limits the strain relaxation depth to the surface, which can be extended along the entire nanorod length (strain still present at the base) by increasing its height up to $\geq 300 \mathrm{~nm}^{25,26}$ We adopted these confirmed dimensions to produce nano-LEDs using a combination of nanosphere lithography (NSL) and reactive ion etching (RIE). ${ }^{27}$ The NSL/RIE procedure has been proven to result in noncoalesced, tightly size-controlled nanorods in contrast to the self-assembled Ni particle mask and selfor catalyst-induced epitaxial growth methods, ${ }^{\mathbf{4 , 1 1 , 1 2 , 2 6}}$ and to be faster, far less equipment demanding than the electron beam lithography and the focused ion beam patterning approaches. ${ }^{\mathbf{1 1 4 , 2 8}}$ Despite the fact that the nano-objects in this work were optically excited and not electrically pumped by means of contacts, they will still be termed nano-LEDs., ${ }^{\mathbf{1 , 2 9 - 3 1}}$ Other commonly used designations for similar nanostructures are QW-nanorods or quantum disc-nanorods or employing the terms nanowires, nanopillars, and nanoposts instead of nanorods. ${ }^{14,28,32-34}$

In the present letter we demonstrate, to the best of our knowledge, for the first time the use of non-resonant microRaman spectroscopy in measuring the strain in individual, wellseparated, and substrate attached InGaN/GaN MQW nanorods based on a clear splitting of the $\mathrm{E}_{2}$ (high) phonon mode of GaN. Giving the simplicity of our new procedure, a statistically relevant study of the strain state in nano-LEDs is performed proving the uniformity of the strain relaxation with regard to the strain state in the initial film and the chosen nanostructuring method. The NSL/RIE nanopatterning enables the controlled formation of nano-emitters perpendicular to the substrate and with homogeneous sizes even at extended defects such as tilt boundaries. We further separate between the effects of strain and variations in thickness and In composition inside the quantum wells by complementary micro-Raman cathodoluminescence (CL) investigations of the same, single nanorod LEDs, not reported so far relying on our literature research. Band profile calculations confirm the optical transitions observed in the CL experiments. Furthermore, microRaman analysis of surface optical (SO) phonon modes of nanoLEDs reveal two peak spectra originating from the anticrossing of axial and planar surface vibrational modes. This paper not only advances the basic understanding of InGaN/GaN MQW nanorods but also validates a potent approach to answer fundamental questions in the rising field of nano-emitters.

\section{Experimental details}

The starting InGaN/GaN MQW films were grown by metalorganic vapor-phase epitaxy (MOVPE) on $c$-plane sapphire substrates. They consist of five pairs of $2.4 \mathrm{~nm}$ thick $\mathrm{In}_{0.18} \mathrm{Ga}_{0.82} \mathrm{~N}$ wells and $14 \mathrm{~nm}$ thick GaN barriers inserted between $130 \mathrm{~nm}$ p-type GaN and $1.6 \mu \mathrm{m}$ n-GaN/2.3 $\mu \mathrm{m}$ undoped GaN. A cross-section scanning electron microscopy (SEM) image of the cleaved film along with the corresponding room temperature panchromatic CL micrograph is shown in Fig. 1a
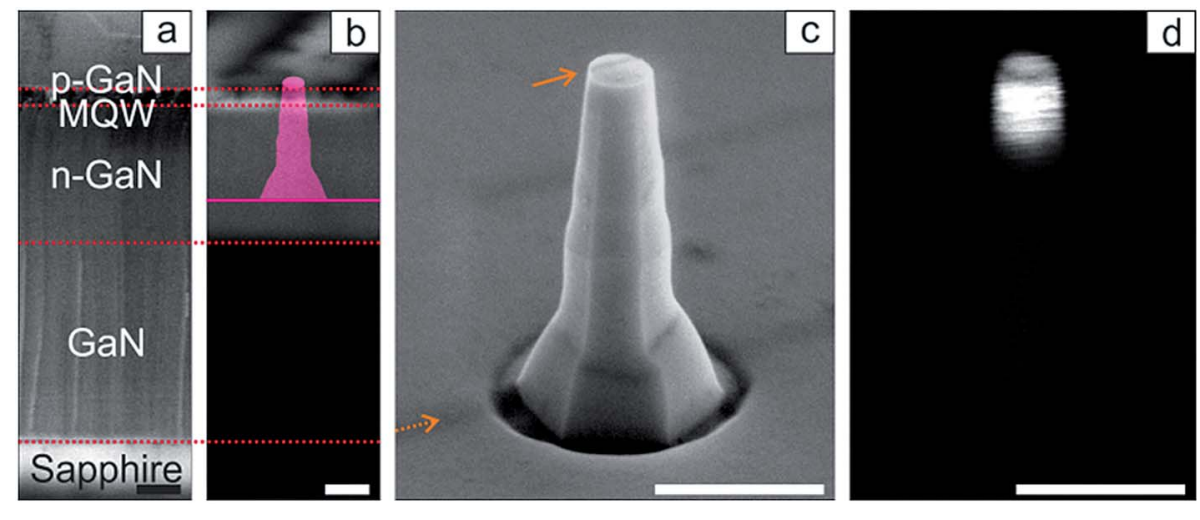

Fig. 1 Cross-section SEM (a) and panchromatic CL (b) images of the starting epitaxial film including a schematic illustration of the etching depth and the remaining GaN film supporting the nanorod in (b). SEM image (c) of an individual isolated nano-LED exhibiting a bright panchromatic CL emission (d) both recorded at a $60^{\circ}$ angle. The arrows in (c) indicate that a tilt boundary crosses this nanorod LED demonstrating the etching homogeneity of the NSL/RIE nanofabrication method irrespective of the growth structure of the initial film. The scale bars are $500 \mathrm{~nm}$. 
and $\mathrm{b}$. The overlying schematic of a nanorod along with the full line illustrates the depth of nanostructuring and the underlying GaN supporting film remained after etching denoted as the GaN substrate. Glass nanospheres with an initial, specified diameter of $490 \pm 20 \mathrm{~nm}$ were spin coated on the film surface to obtain a discontinuous coverage, which is in contrast to the LangmuirBlodgett method that leads to a continuous, densely packed nanosphere monolayer exhibiting high hexagonal periodicity over large areas. ${ }^{27}$ Two plasma steps in a Plasmalab 100 RIE tool from Oxford Instruments were used to reduce the nanosphere diameter $\left(\mathrm{CF}_{4}-\mathrm{H}_{2}-\mathrm{SF}_{6}\right.$ gas mixture, $200 \mathrm{~W} \mathrm{RF}$ (radio frequency), 25 mTorr, 3 minutes) followed by $\mathrm{RIE}\left(\mathrm{Cl}_{2}-\mathrm{Ar}\right.$ gas mixture, $40 \mathrm{~W}$ RF/150 W ICP (inductively coupled plasma), 35 mTorr, 15 minutes) using the dispersed nanospheres as an etching mask to produce single, isolated, and reproducible LED nanorods. A typical SEM example of an individual nano-emitter and its room temperature panchromatic CL emission, both at a $60^{\circ}$ tilt angle, are displayed in Fig. $1 \mathrm{c}$ and $\mathrm{d}$. It can be seen that the NSL/RIE patterning results in slightly tapered cylindrical rods with smooth sidewalls and nanoscale modulation of the surface accompanied by a bright light emission from the quantum well layers at a mean value of $441.08 \pm 5.82 \mathrm{~nm}(2.81 \pm 0.07 \mathrm{eV})$. The nanorods have a mean top diameter of $188.11 \pm 16.92 \mathrm{~nm}$ and a mean height of $1052.54 \pm 43.33 \mathrm{~nm}$ corresponding to a standard deviation of only $9 \%$ (compared with $4 \%$ for the initial nanosphere diameter) and $4 \%$, respectively. These values clearly demonstrate the fine control of nano-LED dimensions that can easily be achieved using the NSL/RIE nanofabrication technique. Moreover, the dry RIE step is not affected by the presence of tilt boundaries characteristic to MOVPE growth of GaN films on sapphire resulting from island-island coalescence before a continuous film forms as proved by the SEM image in Fig. 1c, where a tilt boundary indicated by an arrow runs across this nanorod. ${ }^{35}$

The InGaN/GaN MQW nano-LEDs were experimentally studied at room temperature using a combination of microRaman and CL spectroscopic measurements enabling the understanding of the interplay between strain and optical transitions in individual nano-emitters. The Raman measurements were performed in the backscattering configuration using a LabRam HR800 spectrometer from Horiba Scientific. A linearly polarized laser emitting at $532 \mathrm{~nm}(2.33 \mathrm{eV})$ was used for the Raman excitation. The laser light was tightly focused by a $100 \times$ objective (numerical aperture 0.9 ) resulting in a diameter of the normally incident probing beam of $\sim 0.8 \mu \mathrm{m}$ and in a laser power on the sample surface of $\sim 353 \mu \mathrm{W}$ using a filter corresponding to a power density of only $\sim 70 \mathrm{~kW} \mathrm{~cm}^{-2}$. We observed no damage or structural changes to the nano-LEDs as proved by SEM imaging and CL emission investigations before and after the Raman probing as well as no additional Raman shift due to local heating by the laser beam. The CL spectra were acquired with a fixed (not scanning) electron beam perpendicular to the nanorod top using a GatanMonoCL in a Hitachi S4800 SEM at an acceleration voltage of $5 \mathrm{keV}$ and an emission current of $15 \mu \mathrm{A}$. The same measurement procedure was applied to the epitaxial film, before and after the NSL/RIE patterning, for direct comparison.

\section{Results and discussion}

Two probing configurations schematically shown in Fig. 2a and $\mathrm{b}$ for the micro-Raman technique were employed. We note that two tilt boundaries indicated by arrows meet within this nanorod demonstrating the etching homogeneity and stability regardless of the initial film microstructure. Following the common description for the wurtzite structure the $z$-direction coincides with the $c$-axis of GaN, while the $x$ and $y$ directions correspond to any two perpendicular axes in the $c$-plane of GaN (normal to the $c$-axis). According to the Porto notation, in the $z(x,-) \bar{z}$ scattering geometry the incident laser light propagates in the positive $z$-direction being polarized in the $x$-direction and the backscattered Raman light is detected in the negative $z$-direction so that single nano-LEDs were analyzed from the top,

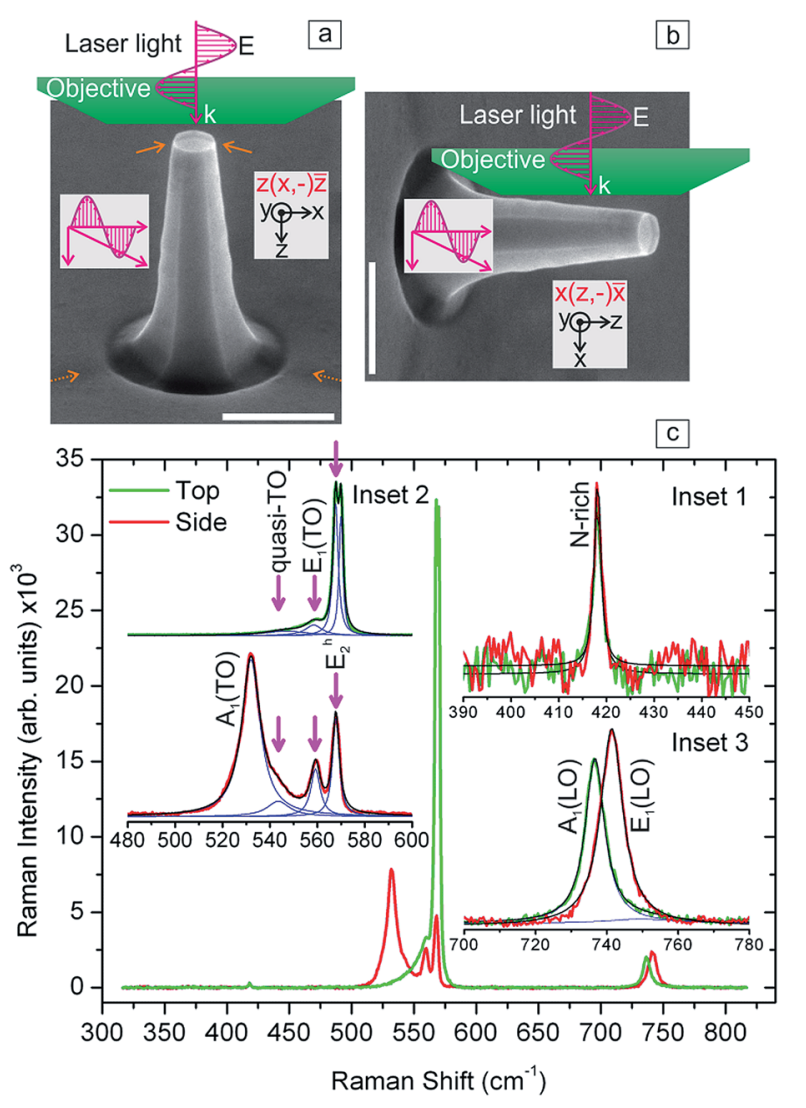

Fig. 2 Micro-Raman measurement geometries used to probe (a) both the nano-LED and the underlying GaN substrate in the top configuration and (b) only the nano-LED in the side configuration. The left insets show the marginal incident light excitations, the right insets describe the Porto notations. Two tilt boundaries intersect in this nanorod LED as pointed out by the arrows in (a). The SEM image was taken before the piranha/ $\mathrm{HCl}$ surface treatments (see also Fig. 4). Complete top and side Raman spectra acquired after the Piranha/ $\mathrm{HCl}$ steps (the SO phonon modes are absent) and their Lorentz fits for three spectral ranges are displayed in (c). The splitting of the $E_{2}^{h}$ Raman peak into two peaks corresponding to the nanorod LED and the GaN substrate in the top geometry (inset 2 - upper graph) demonstrates that the strain state of an individual, isolated nano-LED can separately be analyzed in a non-resonant Raman regime, while still being attached to the substrate. The scale bars are $500 \mathrm{~nm}$. 
whereas in the $x(z,-) \bar{x}$ configuration the probing took place from the side. ${ }^{36}$

Representative, complete Raman spectra measured in the top and side configurations on a nanorod located at the sample edge along with their Lorentz fits are plotted in Fig. 2c. The appearance and intensity of phonon modes mainly depend on the measurement configuration as well as on the marginal, nonparallel light (both in excitation and detection known as the leakage effect) characteristic of the objective-based microRaman technique that leads to a light component perpendicular to the main laser light excitation as described by the left insets in Fig. $2 \mathrm{a}$ and $\mathrm{b} .{ }^{36}$ In the top $z(x,-) \bar{z}$ scattering geometry the selection rules "allow" theoretically only the detection of the $\mathrm{A}_{1}(\mathrm{LO})$ and $\mathrm{E}_{2}$ (high), denoted $\mathrm{E}_{2}^{\mathrm{h}}$, phonon modes associated with the probing configurations $z(x, x) \bar{z}$ and $z(x, y) \bar{z}$, while in the side $x(z,-) \bar{x}$ geometry the "allowed" modes are $\mathrm{A}_{1}(\mathrm{TO})$ and $\mathrm{E}_{1}$ (TO) linked with the configurations $x(z, z) \bar{x}$ and $x(z, y) \bar{x}$ (LO and TO are the acronyms for the longitudinal and transversal optical phonon modes). However, additional phonon modes were observed experimentally: $\mathrm{E}_{1}(\mathrm{TO})$ related to the scattering contribution $x(y, z) \bar{y}$ in the top geometry, $\mathrm{E}_{2}^{\mathrm{h}}$ and $\mathrm{E}_{1}(\mathrm{LO})$ corresponding to the scattering contributions $x(y, y) \bar{x}$ and $x(y, z) \bar{y}$ in the side geometry, as well as the vibration mode of the $\mathrm{N}$-rich Ga-N bond at $\sim 418.13 \mathrm{~cm}^{-1}$ (see inset 1 in Fig. $2 \mathrm{c}$ ) and one unassigned mode at $\sim 543.79 \mathrm{~cm}^{-1}$ (see inset 2 in Fig. 2c) appearing in both measurement geometries. ${ }^{36-38}$ The latter phonon mode was observed exclusively in nanorods (see also Fig. 3a, c and e for the comparison with the film) and it can be attributed to a quasi-TO mode whose frequency lies between the pure $\mathrm{A}_{1}(\mathrm{TO})$ and $\mathrm{E}_{1}(\mathrm{TO})$ mode frequencies. ${ }^{36}$ Since the Raman

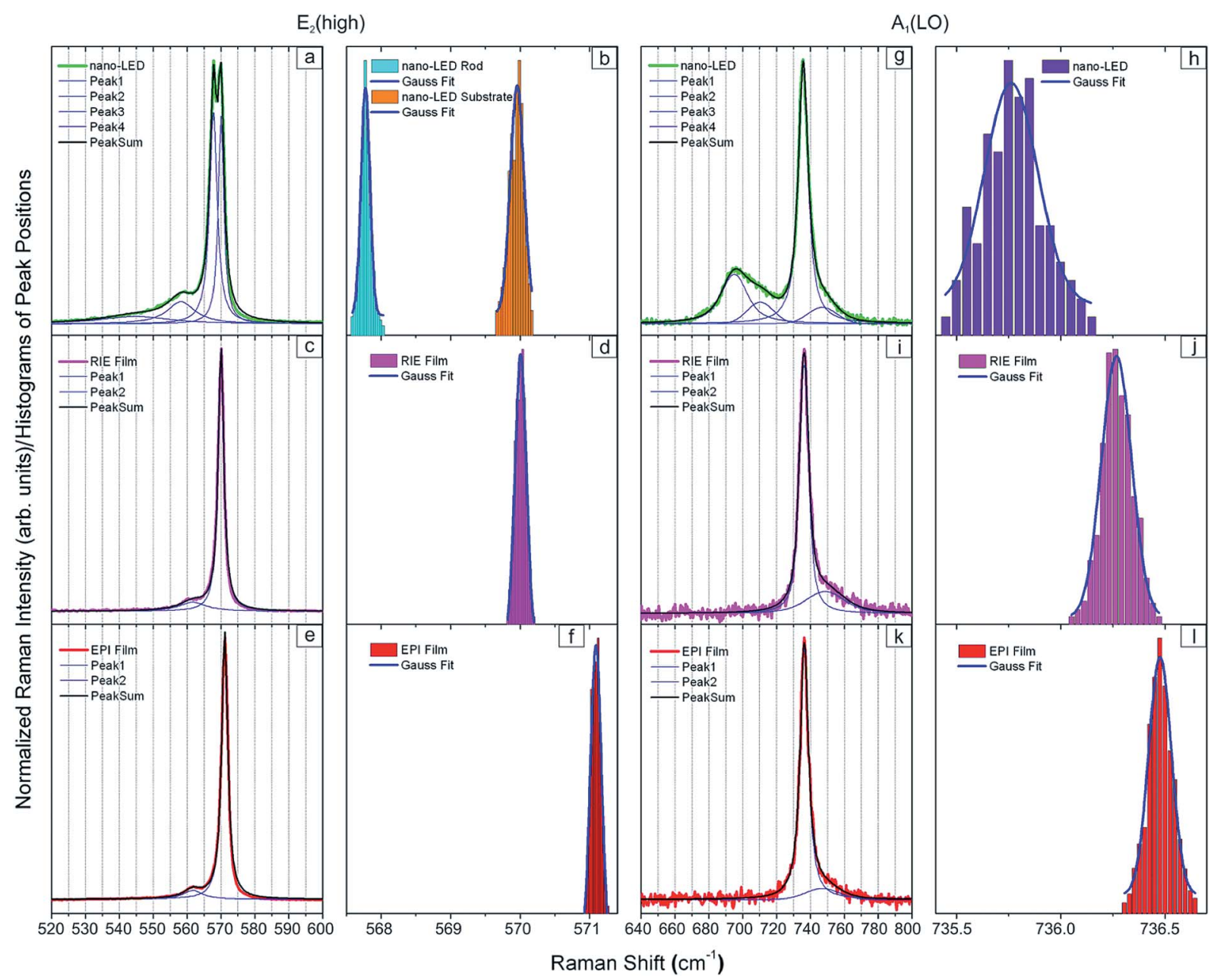

Fig. 3 Partial Lorentz fitted Raman spectra and Gauss fitted peak position histograms of the $E_{2}^{h}(a-f)$ and $A_{1}(L O)(g-l)$ phonon modes for nanoLEDs ( $a, b, g$ and h), etched film without the nanorod LED - RIE film (c, d, i and j), and the starting epitaxial film - EPI film (e, f, k and l). The definite separation between the two histograms in (b) proves the remarkably regular strain relaxation of nano-LEDs with respect to the compressively strained supporting GaN substrate (direct measurements of the substrate are summarized in (d)) and the primary film in (f) that can be achieved through the NSL/RIE nanostructuring procedure. The peaks on the lower frequency side of the $E_{2}^{\text {h }}$ mode are addressed in Fig. 2c, inset 2. In addition to the bulk optical phonons, the nanostructured LEDs show on the lower frequency side of the $A_{1}(L O)$ peak in ( $g$ ) two reproducible surface-related optical phonon modes corresponding to axial and planar vibrations of the surface atoms. The less pronounced Raman shift and the broadening instead of splitting of the $A_{1}(L O)$ mode $(g-l)$ compared with the $E_{2}^{\text {h }}$ mode $(a-f)$ after nano-LED structuring of the epitaxial film are attributed to the less sensitivity of the former to in-plane oscillations of the bulk atoms. The peak on the higher frequency side of the $A_{1}(L O)$ mode visible in ( $\mathrm{g}$, $\mathrm{i}$ and $\mathrm{k}$ ) originates from the sapphire substrate. 
measurements in Fig. 2c were taken after the Piranha/HCl treatments (details are described later in the paper), just the aforementioned bulk phonon modes were present whereas the SO phonon modes were missing (see also Fig. 4e). Their positions are in agreement with the strain-free values for $\mathrm{GaN}^{36}$ $\mathrm{A}_{1}$ (TO) at $\sim 531.99 \mathrm{~cm}^{-1}, \mathrm{E}_{1}(\mathrm{TO})$ at $\sim 558.72 \mathrm{~cm}^{-1}, \mathrm{E}_{2}^{\mathrm{h}}$ nanorods at $\sim 567.75 \mathrm{~cm}^{-1}, \mathrm{~A}_{1}(\mathrm{LO})$ at $\sim 736.68 \mathrm{~cm}^{-1}$ (see inset 3 in Fig. $2 \mathrm{c}$ and the paragraph on the $\mathrm{A}_{1}(\mathrm{LO})$ mode) and $\mathrm{E}_{1}(\mathrm{LO})$ at $\sim 741.38$ $\mathrm{cm}^{-1}$. The use of two probing configurations (laser light being

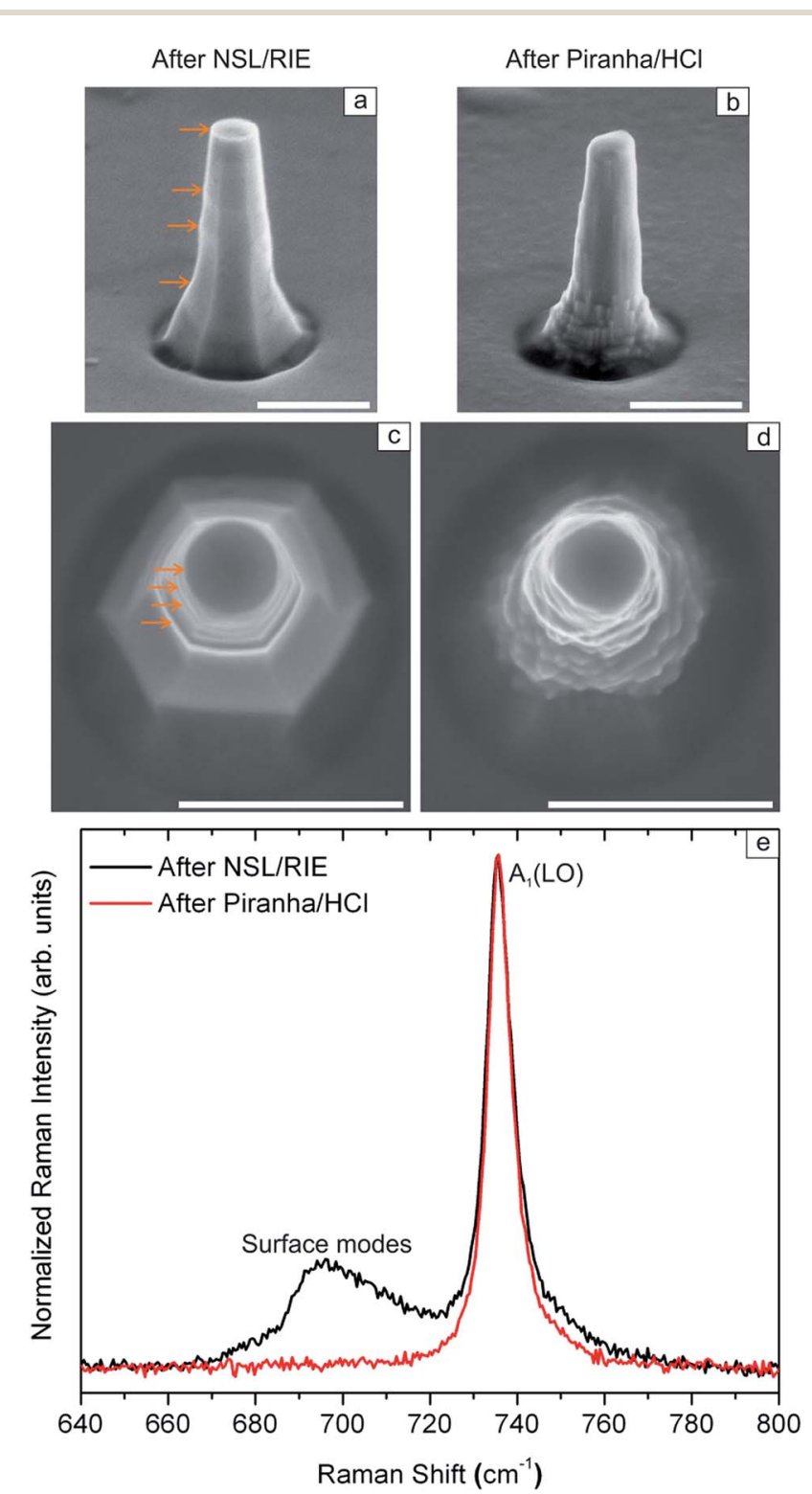

Fig. 4 Side and top SEM images of the same boundary-free nano-LED after NSL/RIE ( $a$ and $c$ ) and piranha/HCl (b and d) steps. The arrows in ( $a$ and $c$ ) highlight the homogeneous surface modulation at the nanoscale initiating the surface optical phonon modes in (e) (see also Fig. 3, panel g). These SO modes disappear when the nanorod LED surface becomes inhomogeneous as in ( $b$ and $d$ ) proving the applicability of Raman spectroscopy to easily distinguish between different surface roughness conditions that can be used to produce tunable phonon assisted LED devices based on nanorods. The scale bars are $500 \mathrm{~nm}$. incident parallel and perpendicular to the nanorod long axis) enabled us not only to explain in detail the Raman spectrum of a nano-LED but also to clearly distinguish between the strain state of a nano-LED and that of the underlying GaN substrate based on the fact that in the side geometry only the nano-LED is measured, while in the top geometry both the nano-LED and the remaining GaN substrate are investigated. It can be clearly seen in the inset 2 of Fig. $2 \mathrm{c}$ that in the latter case the $\mathrm{E}_{2}^{\mathrm{h}}$ Raman peak splits into two peaks whose frequencies correspond to those of the nanorod and the substrate. This finding opens a new possibility to selectively probe a nano-object with a diameter below the diffraction limit, i.e., less than $\lambda / 2$ of the Raman excitation. In other words, the spatial resolution of microRaman becomes the same as the nanorod diameter due to the laser light coupling to the nano-object so that for the first time a nano-LED could be separately probed on the initial substrate consisting of the same material. Because of the limited number of individual nano-LEDs at the sample edge and to present a statistically relevant study, we focus in the following on the Raman results acquired in the top geometry.

Fig. 3a-f show typical, partial Raman spectra fitted with Lorentz functions as well as histograms of the $\mathrm{E}_{2}^{\mathrm{h}}$ peak position based on these fits obtained from separate Raman measurements of 102 single, isolated nano-LEDs and Raman mappings ( 6 maps of $20 \times 20 \mu \mathrm{m}^{2}$ each, step size $0.5 \mu \mathrm{m}$ ) of the film before and after the nanofabrication procedure, all acquired in the $z(x,-) \bar{z}$ scattering geometry. Since the laser excitation $(2.33 \mathrm{eV})$ is below both the band gap of $\mathrm{GaN}(3.41 \mathrm{eV})$ and the MQW emission $(2.81 \mathrm{eV})$, we measured in a non-resonant Raman regime implying only Raman scattering from GaN. ${ }^{36}$ Furthermore, the use of a visible sub-band gap laser ensured that the Raman spectra describe the average strain states over the entire nanorod length and diameter including the nonuniform strain distribution at the nanorod edges as well as over the remaining film thickness. ${ }^{17,39}$ We start our discussion with the distinct splitting of the $\mathrm{E}_{2}^{\mathrm{h}}$ phonon mode in the case of nanostructured LEDs displayed in panel a. The $\mathrm{E}_{2}^{\mathrm{h}}$ two-peak structure is attributed to different strain states of the nanorod (low frequency peak - nano-LED rod) and the GaN substrate, that is, the etched film around the nanorod and below it (high frequency peak nano-LED substrate). The significant separation in the position of the two $\mathrm{E}_{2}^{\mathrm{h}}$ peaks is highly reproducible for all nanorods as demonstrated by the well separated histograms displayed in panel $\mathrm{b}$ with mean values of $567.72 \pm 0.08 \mathrm{~cm}^{-1}$ and $569.95 \pm$ $0.09 \mathrm{~cm}^{-1}$ for nano-LEDs and the substrate, respectively. It is worth noting here that Raman investigations under the same experimental conditions ${ }^{39}$ of a strain-free GaN crystal showed for the $\mathrm{E}_{2}^{\mathrm{h}}$ peak position a mean value of $567.74 \pm 0.07 \mathrm{~cm}^{-1}$, which excellently matches that of nano-LEDs proving the consistent strain relief provided by the NSL/RIE nanostructuring process. One $\mathrm{E}_{2}^{\mathrm{h}}$ peak can be seen in panel $\mathrm{c}$ when mapping the etched film without nanorods (RIE Film) occurring at a similar frequency corresponding to a similar strain level as the right peak in panel a. The corresponding histogram in panel $\mathrm{d}$ exhibits a mean value of $570.01 \pm 0.07 \mathrm{~cm}^{-1}$ that fits very well with that of the substrate in panel b. This demonstrates the capability of micro-Raman spectroscopy to plainly, quickly, and 
unambiguously assess the strain state of single nano-emitters attached to the initial substrate even without the need for a resonant laser excitation. The mapping of the starting strained film (EPI Film) also shows one $\mathrm{E}_{2}^{\mathrm{h}}$ peak located at a higher frequency associated with a higher strain level resulting in a histogram with a mean value of $571.09 \pm 0.06 \mathrm{~cm}^{-1}$ as plotted in panels e and $\mathrm{f}$, respectively. As expected, the originally compressively strained InGaN/GaN MQW epilayers relaxed through transformation into nanorods due to the formation of free surface areas and high-aspect ratios. ${ }^{\mathbf{1 2 , 1 4 , 2 5 , 2 6}}$

In the present research, a mean Raman shift of $3.37 \pm 0.02$ $\mathrm{cm}^{-1}$ is determined as the difference between the mean values of statistically significant Raman datasets shown in Fig. $3 \mathrm{~b}$ (nano-LED rod histogram) and Fig. 3f (EPI film). This shift results in a mean in-plane strain of $\varepsilon_{||}=(-29.36 \pm 0.17) \times 10^{-4}$ or $-0.29 \%$ that was incorporated in the film during the cooling to room temperature after the epitaxial growth procedure at elevated temperatures had taken place (see eqn (1) in the ESI $\dagger$ ). The strain has been caused by the different thermal expansion coefficients of the sapphire substrate and the GaN layer as well as the lattice mismatch between the InGaN/GaN quantum layers and finally relaxed as a consequence of nanorod formation that gives rise to freely expanding lattices at free surfaces. ${ }^{18,26}$

We obtained an in-plane strain of $-2.27 \%$ for the initial film that decreases to $-1.97 \%$ for the nanorods using $a_{\mathrm{NR}}=3.189 \AA$ that equals the lattice constant of strain-free GaN in agreement with the Raman results, the calculated $a_{\text {Epi }}=3.179 \AA$, and $a_{\text {InGaN }}$ $=3.25 \AA$ for $x=0.18$ In (see eqn (2) and (3) in the ESI $\dagger$ ). The difference of $-0.30 \%$ for the QWs is very close to the strain relaxation value of $-0.29 \%$ calculated for GaN from the Raman data. Thus, even in a non-resonant Raman regime, one can indirectly estimate the strain state in the InGaN/GaN MQWs only based on the Raman signal from GaN. It should be noted that a uniform strain state was assumed in these calculations as applied to a QW film. The simulation of the radial strain distribution in a QW-nanorod geometry using the nextnano3 $\operatorname{code}^{\mathbf{4 0}}$ gives a weighted mean in-plane strain of $\varepsilon_{||}=-1.78 \%$ when averaging over the entire QW region (for more details see ESI, Fig. S1 $\dagger$ ). As expected, this value is smaller than $-1.97 \%$ because of including the strain relaxation at the nanorod free surface areas. The strain difference of only $-0.19 \%$ corresponds to an overestimation of $9.6 \%$ for the average in-plane strain amount in the film with respect to that in the nanorod. This effect becomes significant when the strain-relaxed region inside the nano-emitter approaches its diameter. ${ }^{\mathbf{2 9 , 4 1 - 4 4}}$

Next, we applied a similar data analysis to the same Raman spectra with respect to the $\mathrm{A}_{1}(\mathrm{LO})$ phonon mode. The results are summarized in Fig. $3 \mathrm{~g}-\mathrm{l}$. In contrast to the two-peak structure of the $E_{2}^{\mathrm{h}}$ mode of nano-LEDs, the $\mathrm{A}_{1}(\mathrm{LO})$ mode consists of one peak as shown in panel $\mathrm{g}$ with an average position value of $735.79 \pm 0.14 \mathrm{~cm}^{-1}$ determined from the histogram in panel $\mathrm{h}$. This value differs from that of $733.95 \pm 0.05 \mathrm{~cm}^{-1}$ obtained under the same experimental conditions on a strain-free $\mathrm{GaN}$ crystal indicating that the $\mathrm{A}_{1}(\mathrm{LO})$ peak includes besides the Raman scattering from the nanorod, the Raman signal from the underlying GaN substrate. The mixture of these two contributions is further supported by the larger width of the nano-LED histogram in panel $h$ as compared with the widths of the film histograms after and before etching displayed in panel $\mathrm{j}$ and 1 with mean position values of $736.26 \pm 0.12 \mathrm{~cm}^{-1}$ and $736.47 \pm 0.10 \mathrm{~cm}^{-1}$, respectively. As evidenced by these histograms, a shift towards lower frequencies was measured for the $\mathrm{A}_{1}$ (LO) mode when structuring the starting film into nanorods due to the strain relaxation (for further details see ESI $\dagger$ ).

Noticeable in this spectral region for the nano-emitters is the coexistence of the $\mathrm{A}_{1}(\mathrm{LO})$ bulk optical phonons with the surface optical phonons visible on the lower frequency side of the $\mathrm{A}_{1}(\mathrm{LO})$ peak as displayed in panel $\mathrm{g}$ of Fig. 3. The consistent fitting of the SO mode in two well resolved peaks with mean position values of $694.24 \pm 1.91 \mathrm{~cm}^{-1}$ and $709.10 \pm 1.32 \mathrm{~cm}^{-1}$ is the result of the anticrossing between two oscillation modes of the surface atoms known as Fröhlich modes, namely parallel (axial) and perpendicular (planar) to the $c$-axis ( $z$-direction) of the rods with $\mathrm{A}_{1}$ and $\mathrm{E}_{1}$ symmetry, respectively. ${ }^{22,23}$ The Raman sensitivity to both symmetries in the top measurement geometry for the surface vibrations, similar to the bulk ones, is due to the simultaneous laser excitation of the top and sidewalls of the nanorod as described in Fig. 2a and c. In our case, the SO phonons are activated by the regular surface modulation at the nanoscale indicated by the arrows on side and top SEM images of a representative and boundary-free rod in Fig. 4a and c. This modulation induces the breakdown of the translational symmetry of the SO potential creating a larger wave vector along the surface so that the SO mode frequencies are markedly separated from those of the bulk. ${ }^{20,28,37}$ In addition to the nanoscale surface modulation, it is important to point out that our vertically and tightly size-controlled nano-LEDs ensure the same orientation of the rod $c$-axis with respect to the incident light and a constant filling factor of $0.31 \pm 0.04$ (rod to air area ratio within the laser beam probing diameter). ${ }^{22,23}$ By keeping these three features mainly fixed through NSL/RIE nanofabrication, we demonstrated the control of the sO mode characteristics such as splitting or symmetry mixing between the axial and planar surface-related phonon modes and their frequencies within small standard deviation values.

The nanorod surface was further modified at the nanoscale level using a combined process consisting of a cleaning step in a piranha solution $\left(\mathrm{H}_{2} \mathrm{O}_{2} 30 \%-\mathrm{H}_{2} \mathrm{SO}_{4} 96 \%\right.$ mixture, ratio $1: 2,30$ minutes) to eliminate organic residues followed by an etching step in an $\mathrm{HCl}$ solution $\left(\mathrm{HCl} 32 \%-\mathrm{H}_{2} \mathrm{O}\right.$ mixture, ratio $1: 1$, boiling, 2 minutes) to remove the NSL/RIE damage. The effect is evident in Fig. 4 when comparing the SEM images of the same nanorod before (panel a and c) and after (panel b and d) these treatments as well as the typical, partial Raman spectra (panel e). The change in the nanorod morphology not only increases the surface roughness but also decreases the effective optical cross-section exposed to the probing laser beam due to the geometry modification and size reduction. Interestingly, the SO phonon peak vanished despite the common belief that the surface roughness, which increases because of the irregular surface modulation of the nanorods after the piranha/ $\mathrm{HCl}$ steps, should lead to the occurrence of SO modes because of the surface symmetry breaking. ${ }^{28,37}$ This suggests that the SO mode 
intensity may be the result of the competition between the decrease caused by the geometry/size changes and the increase caused by the induced surface roughness. Our findings validate the straightforward application of Raman spectroscopy in the case of nano-objects composed of anisotropic polar semiconductors to differentiate between distinct types of surface roughnesses related to different degrees of surface faceting and smoothing, which unexpectedly do not necessarily always lead to the appearance of SO modes. Furthermore, it confirms that the assignment of these phonon modes to the surface is accurate, while the bulk phonon modes, located at their strain-free positions as shown in Fig. 2c, are not affected by the surface alteration. It is worth noting here that the nano-emitters radiate light virtually at the same wavelength after the nanoscale modification of their surface. These results are also of great relevance for the future design of nano-LED devices. From the electrical performance point of view, such a nanoscale control of the SO phonons may provide an unexplored approach to use the Fröhlich phonon-electron coupling for tuning the interaction of phonons with electrons and thus the electrical transport in these nanostructures. ${ }^{22,23}$ With respect to the light emission efficiency, an appropriate surface processing may provide a tradeoff between the removal of defects and thus the gain in the internal quantum efficiency and the preservation of the SO phonon modes.

To get a deeper insight into the interplay between strain and light emission of nano-LEDs, we accomplished a complementary micro-Raman - CL spectroscopic study on the same, isolated nanorods supported by band profile calculations. It should be stressed that the micro-Raman and CL detection areas are of the order of the nanorod diameter so that the two datasets are comparable. Since the strain relaxation and consequently the emission depend on the diameter and height of the nanorods, our tightly size-controlled nano-LEDs are suitable for this study. The strain relaxation proved by microRaman spectroscopy on GaN is accompanied by the reduction of the in-plane strain in InGaN/GaN MQWs as calculated earlier in the paper. This translates further into a decrease of the strain-induced piezoelectric field, which in turn lowers the quantum confined Stark effect and consequently blue-shifts the QW emission wavelength of nano-emitters with respect to that of the epitaxial film..$^{\mathbf{1 4 1 8 , 1 9}}$ Fig. 5a displays two typical CL spectra documenting this effect. A blue-shift caused by the band filling can be ruled out based on CL measurements carried out with increasing SEM emission currents ranging from $5 \mu \mathrm{A}$ to $20 \mu \mathrm{A}$ (for the data see ESI, Fig. S2 $\dagger$ ). Despite several publications on this fundamental aspect, there is no paper, to the best of our knowledge, reporting a full experimental strain - emission dependence analysis as that presented in Fig. 5b. Here, thirty representative rods covering the entire range of strain states ( $\mathrm{E}_{2}^{\mathrm{h}}$ peak positions) in the nano-LED rod histogram of Fig. $3 \mathrm{~b}$ have been selected. The slightly different strain levels are attributed to the presence of defects such as dislocations already present in the GaN epilayer, which give rise to the dark CL contrast regions on the upper, planar area in Fig. 1b, while a similar defect density is considered to be induced by the RIE process at the surface of all nanorods. However, it has been

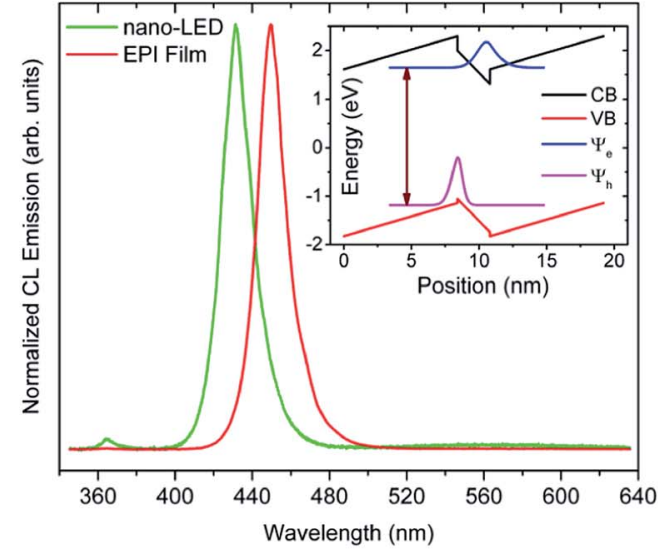

a
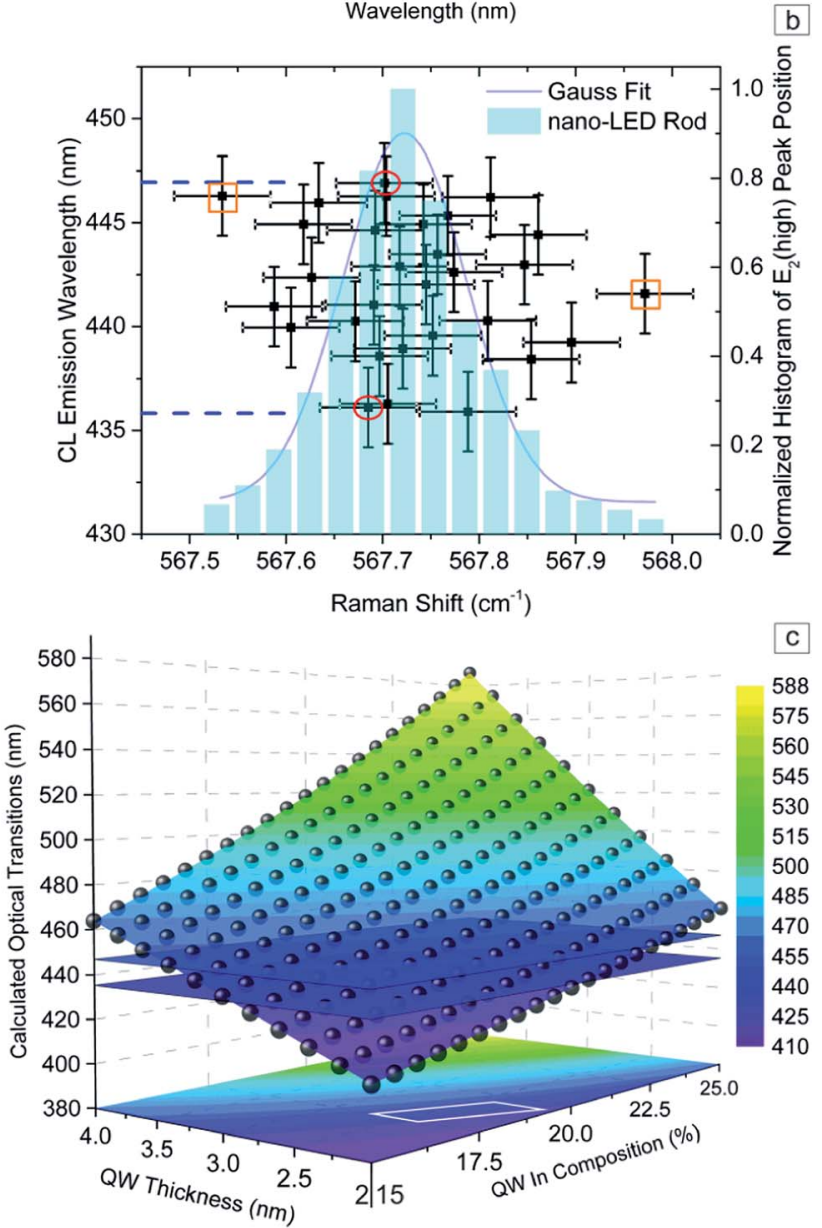

Fig. 5 The CL spectra (a) evidence the blue shift of the QW emission wavelength of nano-emitters in relation to that of the starting epitaxial film. This confirms the strain relaxation in the nanostructured LEDs measured by the micro-Raman technique. Correlative strain - emission data (b) obtained from the same nanorods chosen to overlay with the $E_{2}^{\mathrm{h}}$ peak position nano-LED rod histogram in Fig. 3b. The points marked by squares indicate the outermost detected strain states, while the exemplary points highlighted by circles correspond to two nanoLEDs being in the same strain state but showing distinct radiative transition wavelengths ascribed to local QW thickness and In fraction fluctuations. Band profile calculations (see the inset in (a)) reveal the effect of these nanoscale variations on the optical transitions plotted in (c). The crossing between the two experimental planes coinciding with the dashed lines in (b) and the calculated 3D surface results in a rectangular projection around the growth values, which defines quantitatively the levels of thickness and In content deviations. 
shown that the stress fields of dislocations can cancel partially, totally or not at all as a result of their interaction to minimize the strain energy. ${ }^{45}$ Because the positions of the Raman peaks remain virtually unchanged before and after the piranha/ $\mathrm{HCl}$ surface treatments, it is concluded that the formation and interaction of dislocations play a minor role in the strain relaxation in nanorods that occurs mainly by means of nanostructuring. The mean values evaluated for these nano-LEDs and thirty CL measurements of the unpatterned film are 441.08 $\pm 5.82 \mathrm{~nm}(2.81 \pm 0.07 \mathrm{eV})$ and $447.66 \pm 1.92 \mathrm{~nm}(2.77 \pm 0.02$ $\mathrm{eV})$, respectively. In the literature, the scattering in the $\mathrm{QW}$ emission wavelength of nanorods is generally attributed to local variations in strain, thickness, and In content. ${ }^{14-16,21,34,46,47}$ These nanoscale fluctuations in the QW region are more accessible when exciting CL in nano-LEDs as compared with the epitaxial film because the collection is limited only to the nanorod volume, while being averaged over a larger volume in the film. ${ }^{48}$ Interestingly, the same strain level $\left( \pm 0.05 \mathrm{~cm}^{-1}\right)$ does not necessarily result in the same QW emission wavelength $( \pm 1.92$ $\mathrm{nm}$ ) for all nanorods. For example, the difference in the CL emission for the two nanorods with similar strain states marked by circles in panel b is $10.81 \mathrm{~nm}$ (69 meV), which can only be assigned to locally different quantum well thicknesses and In concentrations. Although such intrinsic fluctuations in the QW layers were observed, their effect on the light emission could not unambiguously be separated from that of the strain contribution so far..$^{20,21,34,46}$

Theoretical calculations using the nextnano3 $\operatorname{code}^{40}$ were performed to further understand the impact of the QW structural properties on the main optical transition energy in nanoLEDs (i.e., between the first energy states in the QW conduction and valance bands as illustrated by the arrow in the inset of Fig. 5a, where the hole wavefunctions were obtained based on the $6 \times 6 \mathbf{k} \cdot \mathbf{p}$ method, while the electron wavefunctions utilizing the effective mass approximation). The surface band bending was neglected because of the large diameter of our nanorods. Since the excitons tend to dissociate at higher temperatures and the CL measurements were taken at room temperature, the exciton binding energy was not included. ${ }^{49} \mathrm{We}$ simulated an infinite array of InGaN quantum wells and GaN barrier layers as a function of the QW thickness (2-4 nm, every $0.2 \mathrm{~nm}$ ) and In content (15-25\%, every $0.5 \%$ ) using $a_{\mathrm{GaN}}=3.189$ $\AA$ in agreement with the Raman results and taking the spontaneous and strain-induced piezoelectric polarization fields into account (for more details see ESI, Fig. S3†). An example of a computed band structure with a nominal QW thickness of $2.4 \mathrm{~nm}$ and an In content of $18 \%$ can be seen in the inset of Fig. 5a, which gives an emission at $438.09 \mathrm{~nm}$ that compares very well with the measured emission at around $441.08 \pm 5.82$ $\mathrm{nm}$ indicating the reliability of the simulations. The same procedure was applied to unravel the effect of strain (Raman shift) dispersion in Fig. 5b on the QW emission wavelength of individual nano-emitters. Using the eqn (1) and (2) in the ESI, $\uparrow$ the GaN lattice constants for the two nanorods (marked by squares in panel b) corresponding to the outermost detected strain states were found to be $3.18829 \AA$ and $3.18951 \AA$. The mean difference in their emission position with respect to
$a_{\mathrm{GaN}}=3.189 \AA$ is only $0.55 \mathrm{~nm}$ proving that the strain-emission variations from nano-LED to nano-LED are negligible in our tightly size-controlled nanorods (see ESI, Fig. S3†). This point further confirms that the measured variations in the QW emission position originate from nonuniform QW widths and In contents. Fig. 5c shows the 3D plot extracted from the calculated QW emission wavelengths intersected by two planes delimiting the range of experimental data corresponding to the horizontal dashed lines in panel b. This comparison provides a quantitative estimation of the QW thickness and In composition uniformity between 2.07-2.72 $\mathrm{nm}$ and 17.5-19.5\%, respectively highlighted by the white rectangular projection in panel c. Details on obtaining the limits of this rectangle are given in the ESI, Fig. S4.† These variations are small as compared to recent nanoscale imaging experiments on InGaN/ GaN QW nano-objects grown by MOVPE. ${ }^{\mathbf{2 0 , 2 1}}$ It proves that even slight irregularities inherent to the epitaxial growth can be quantified using the novel methodology employed in the present paper, thereby providing a unique and efficient route for the examination and optimization of technologically relevant semiconductor nanorod-based emitters.

\section{Conclusions}

In summary, we have used a combination of nanosphere lithography and reactive ion etching of epitaxial InGaN/GaN MQW layers on sapphire substrates to produce tightly sizecontrolled nano-LEDs with smartly chosen dimensions. Nonresonant micro-Raman spectroscopy has been employed to differentiate between the Raman signals originating from a single, diffraction-limited nanorod LED and the supporting substrate based on a distinct two-peak structure for the in-plane strain sensitive $\mathrm{E}_{2}$ (high) phonon mode of GaN. We observed a remarkably consistent strain relaxation for the nanostructured LEDs with respect to the compressive strain in the initial epitaxial film and the used nanofabrication procedure. Complementary micro-Raman - CL investigations of the same, individual nano-LEDs corroborated by band profile calculations showed that the scattering in the QW emission wavelength of nano-emitters with equivalent strain conditions is caused by the local variations in the QW thickness and the In composition. Moreover, we demonstrated that by modifying the nanorod surface roughness, the occurrence of surface optical phonon modes can be tuned without affecting the strain states and QW peak emissions, thereby opening an intriguing perspective for nano-tailoring the properties of phonon assisted nanorod LED devices. These results demonstrate the full potential of the correlative experimental and theoretical approach adopted herein for a detailed structural and optical characterization of single nano-emitters that can be extended to other materials containing quantum dots or quantum wells with tunable emission over the entire visible range.

\section{Acknowledgements}

The research leading to these results has received funding from the European Union Seventh Framework Programme 
[FP7/2007-2013] under grant agreement number 280566, project "UnivSEM" and from the German Research Foundation (DFG) within the project "Dynamics and Interactions of Semiconductor Nanowires for Optoelectronics (FOR 1616)". M. L. thanks the DFG for financial support through the Cluster of Excellence "Engineering of Advanced Materials" at the University of Erlangen-Nuremberg. The authors gratefully acknowledge Aixtron SE for providing the epitaxial films.

\section{References}

1 M.-L. Kuo, Y.-S. Kim, M.-L. Hsieh and S.-Y. Lin, Nano Lett., 2010, 11, 476-481.

2 X. Hugon, P. Gilet and P. Mottier, Semiconductor Today, 2011, 6, 84-86.

3 H.-W. Lin, Y.-J. Lu, H.-Y. Chen, H.-M. Lee and S. Gwo, Appl. Phys. Lett., 2010, 97, 073101-073103.

4 R. W. Cole and J. N. Turner, Microsc. Microanal., 2008, 14, 243-250.

5 F. Qian, Y. Li, S. Gradecak, H.-G. Park, Y. Dong, Y. Ding, Z. L. Wang and C. M. Lieber, Nat. Mater., 2008, 7, 701-706.

6 Y.-J. Lu, H.-W. Lin, H.-Y. Chen, Y.-C. Yang and S. Gwo, Appl. Phys. Lett., 2011, 98, 233101.

7 S. L. Howell, S. Padalkar, K. Yoon, Q. Li, D. D. Koleske, J. J. Wierer, G. T. Wang and L. J. Lauhon, Nano Lett., 2013, 13, 5123-5128.

8 J.-H. Park, M.-H. Kim, S. Kissinger and C.-R. Lee, Nanoscale, 2013, 5, 2959-2966.

9 C.-H. Ho, D.-H. Lien, H.-C. Chang, C.-A. Lin, C.-F. Kang, M.-K. Hsing, K.-Y. Lai and J.-H. He, Nanoscale, 2012, 4, 7346-7349.

10 S. Deshpande, J. Heo, A. Das and P. Bhattacharya, Nat. Commun., 2013, 4, 1675.

11 J. Bai, Q. Wang and T. Wang, J. Appl. Phys., 2012, 111, 113103-113107.

12 Q. Wang, J. Bai, Y. P. Gong and T. Wang, J. Phys. D: Appl. Phys., 2011, 44, 395102.

13 L.-Y. Chen, Y.-Y. Huang, C.-H. Chang, Y.-H. Sun, Y.-W. Cheng, M.-Y. Ke, C.-P. Chen and J. Huang, Opt. Express, 2010, 18, 7664-7669.

14 C. Horng-Shyang, Y. Dong-Ming, L. Yen-Cheng, C. ChengYen, H. Chi-Feng, T. Tsung-Yi, C. C. Yang, W. Cen-Shawn and C. Chii-Dong, Nanotechnology, 2006, 17, 1454.

15 F. Limbach, C. Hauswald, J. Lähnemann, M. Wölz, O. Brandt, A. Trampert, M. Hanke, U. Jahn, R. Calarco, L. Geelhaar and H. Riechert, Nanotechnology, 2012, 23, 465301.

16 A. L. Bavencove, G. Tourbot, J. Garcia, Y. Désières, P. Gilet, F. Levy, B. André, B. Gayral, B. Daudin and D. Le Si, Nanotechnology, 2011, 22, 345705.

17 M. Wölz, M. Ramsteiner, V. M. Kaganer, O. Brandt, L. Geelhaar and H. Riechert, Nano Lett., 2013, 13, 4053-4059.

18 V. D. Jovanović and P. Harrison, Strained Quantum Wells, in Quantum Wells, Wires and Dots, John Wiley \& Sons, Ltd, 2006, pp. 219-241.

19 U. M. E. Christmas, A. D. Andreev and D. A. Faux, J. Appl. Phys., 2005, 98, 073522.
20 E. Poliani, M. R. Wagner, J. S. Reparaz, M. Mandl, M. Strassburg, X. Kong, A. Trampert, C. M. Sotomayor Torres, A. Hoffmann and J. Maultzsch, Nano Lett., 2013, 13, 3205-3212.

21 L. Rigutti, I. Blum, D. Shinde, D. Hernández-Maldonado, W. Lefebvre, J. Houard, F. Vurpillot, A. Vella, M. Tchernycheva, C. Durand, J. Eymery and B. Deconihout, Nano Lett., 2013, 14, 107-114.

22 R. Mata, A. Cros, K. Hestroffer and B. Daudin, Phys. Rev. B: Condens. Matter Mater. Phys., 2012, 85, 035322.

23 J. Wang, F. Demangeot, R. Péchou, A. Ponchet, A. Cros and B. Daudin, Phys. Rev. B: Condens. Matter Mater. Phys., 2012, 85, 155432.

24 Y.-H. Hsiao, C.-Y. Chen, L.-C. Huang, G.-J. Lin, D.-H. Lien, J.-J. Huang and J.-H. He, Nanoscale, 2014, 6, 2624-2628.

25 W. J. Tseng, M. Gonzalez, L. Dillemans, K. Cheng, S. J. Jiang, P. M. Vereecken, G. Borghs and R. R. Lieten, Appl. Phys. Lett., 2012, 101, 253102.

26 M. Hugues, P. A. Shields, F. Sacconi, M. Mexis, M. Auf der Maur, M. Cooke, M. Dineen, A. Di Carlo, D. W. E. Allsopp and J. Zúñiga-Pérez, J. Appl. Physiol., 2013, 114, 084307.

27 S. W. Schmitt, F. Schechtel, D. Amkreutz, M. Bashouti, S. K. Srivastava, B. Hoffmann, C. Dieker, E. Spiecker, B. Rech and S. H. Christiansen, Nano Lett., 2012, 12, 40504054.

28 X. H. Wang, J. Q. Ning, S. J. Xu and H. W. Choi, J. Appl. Phys., 2011, 110, 093111.

29 W. Yuh-Renn, C. Chinghua, C. Cheng-Yu, Y. Peichen and K. Hao-Chung, IEEE J. Sel. Top. Quantum Electron., 2009, 15, 1226-1233.

30 Q. Li, K. R. Westlake, M. H. Crawford, S. R. Lee, D. D. K1oleske, J. J. Figiel, K. C. Cross, S. Fathololoumi, Z. Mi and G. T. Wang, Opt. Express, 2011, 19, 25528-25534.

31 J. R. Riley, S. Padalkar, Q. Li, P. Lu, D. D. Koleske, J. J. Wierer, G. T. Wang and L. J. Lauhon, Nano Lett., 2013, 13, 4317-4325. 32 C. Rivera, U. Jahn, T. Flissikowski, J. L. Pau, E. Muñoz and H. T. Grahn, Phys. Rev. B: Condens. Matter Mater. Phys., 2007, 75, 045316.

33 L. F. Zagonel, S. Mazzucco, M. Tencé, K. March, R. Bernard, B. t. Laslier, G. n. Jacopin, M. Tchernycheva, L. Rigutti, F. H. Julien, R. Songmuang and M. Kociak, Nano Lett., 2010, 11, 568-573.

34 G. Tourbot, C. Bougerol, F. Glas, L. F. Zagonel, Z. Mahfoud, S. Meuret, P. Gilet, M. Kociak, B. Gayral and B. Daudin, Nanotechnology, 2012, 23, 135703.

35 G. Pierre, Rep. Prog. Phys., 2004, 67, 667.

36 H. Hiroshi, J. Phys.: Condens. Matter, 2002, 14, R967.

37 J. H. Zhu, J. Q. Ning, C. C. Zheng, S. J. Xu, S. M. Zhang and H. Yang, Appl. Phys. Lett., 2011, 99, 113115.

38 J. Q. Ning, S. J. Xu, D. P. Yu, Y. Y. Shan and S. T. Lee, Appl. Phys. Lett., 2007, 91, 103117.

39 S. Choi, E. Heller, D. Dorsey, R. Vetury and S. Graham, J. Appl. Phys., 2013, 113, 093510.

40 See http://www.nextnano.de/nextnano3 for the program.

41 J. Renard, R. Songmuang, G. Tourbot, C. Bougerol, B. Daudin and B. Gayral, Phys. Rev. B: Condens. Matter Mater. Phys., 2009, 80, 121305. 
42 F. Furtmayr, J. Teubert, P. Becker, S. Conesa-Boj, J. R. Morante, A. Chernikov, S. Schäfer, S. Chatterjee, J. Arbiol and M. Eickhoff, Phys. Rev. B: Condens. Matter Mater. Phys., 2011, 84, 205303.

43 G. Jacopin, L. Rigutti, J. Teubert, F. H. Julien, F. Furtmayr, P. Komninou, T. Kehagias, M. Eickhoff and M. Tchernycheva, Nanotechnology, 2013, 24, 125201.

44 P. Yu, C. H. Chiu, Y.-R. Wu, H. H. Yen, J. R. Chen, C. C. Kao, H.-W. Yang, H. C. Kuo, T. C. Lu, W. Y. Yeh and S. C. Wang, Appl. Phys. Lett., 2008, 93, 081110.

45 G. Sarau, S. Christiansen, M. Holla and W. Seifert, Sol. Energy Mater. Sol. Cells, 2011, 95, 2264-2271.
46 J. Lähnemann, O. Brandt, C. Pfüller, T. Flissikowski, U. Jahn, E. Luna, M. Hanke, M. Knelangen, A. Trampert and H. T. Grahn, Phys. Rev. B: Condens. Matter Mater. Phys., 2011, 84, 155303.

47 M. Wölz, J. Lähnemann, O. Brandt, V. M. Kaganer, M. Ramsteiner, C. Pfüller, C. Hauswald, C. N. Huang, L. Geelhaar and H. Riechert, Nanotechnology, 2012, 23, 455203.

48 B. Wilsch, U. Jahn, B. Jenichen, J. Lähnemann, H. T. Grahn, H. Wang and H. Yang, Appl. Phys. Lett., 2013, 102, 052109.

49 V. D. Jovanović and P. Harrison, Strained Quantum Wells, in Quantum Wells, Wires and Dots, John Wiley \& Sons, Ltd, 2006, p. 190. 